\title{
Brazilian scientific production on herbal medicines used in dentistry
}

CASTRO, R.D..,1, OLIVEIRA, J.A. ${ }^{2}$, VASCONCELOS, L.C. ${ }^{2}$, MACIEL, P.P. ${ }^{2}$, BRASIL, V.L.M. ${ }^{2}$

1 Professor Doutor do Programa de Pós-graduação em Odontologia da UFPB, Universidade Federal da Paraíba, Campus I, Cidade universitária, 58051-900, João Pessoa, PB, Brasil; ' Programa de Pós-graduação em Odontologia da UFPB, Universidade Federal da Paraíba, Campus I, Cidade universitária, 58051-900, João Pessoa, PB, Brasil. *Autor correspondente: ricardodiasdecastro@yahoo.com.br.

\begin{abstract}
The objective of this study was to critically analyze the scientific production published in specialized Brazilian journals concerning the use of medicinal plants in dentistry. A literature review was carried out using an indirect documentation technique by means of a bibliographical study. Four examiners performed independent searches in Brazilian journals of medicinal plants indexed in the database SciELO (Brazilian Journal of Pharmacognosy; Brazilian Journal of Medicinal Plants; Brazilian Journal of Pharmaceutical Sciences; and Acta Botanica Brasilica) using the descriptors "herbal medicine/phytotherapy" or "medicinal plants" and "dentistry ". The articles published from 2002 to 2012 addressing the use of medicinal plants in dentistry were included and analyzed. The searches based on the descriptors and reading of abstracts, resulted in 155 articles. Of these, 44 were read in full and a total of 16 publications met the eligibility criteria and were selected. Laboratory studies predominated (10) and were limited to the evaluation of antimicrobial properties by means of tests for determining inhibitory, fungicidal and bactericidal concentrations. Three literature reviews and only one clinical trial with no blinding and randomization were found. It is highlighted the need for better methodological designs in the researches and greater production of clinical or in vivo studies.
\end{abstract}

Key words: Dentistry, Ethnobotany, Medicinal Plants, Phytotherapy, Plant Extracts.

RESUMO: Produção científica brasileira sobre o uso de plantas medicinais na odontologia. O objetivo deste estudo foi analisar criticamente a produção científica acerca do uso de plantas medicinais em Odontologia nos periódicos brasileiros especializados. Realizou-se uma revisão da literatura, utilizando técnica de documentação indireta a partir de um estudo bibliográfico. Quatro pesquisadores procederam, de forma independente, uma busca nas revistas brasileiras de plantas medicinais indexadas na base de dados Scielo (Revista brasileira de farmacognosia; Revista brasileira de plantas medicinais; Brazilian journal of pharmaceutical sciences; Acta botânica. brasílica) utilizando como descritores "fitoterapia" ou "plantas medicinais" e "Odontologia". Os artigos publicados de 2002 a 2012 que envolveram o uso de plantas medicinais em Odontologia foram incluídos e analisados. A busca por descritores e leitura de resumos gerou 155 artigos, dos quais 44 foram lidos na íntegra, sendo selecionadas 16 publicações que se enquadraram aos critérios requeridos. Estudos laboratoriais foram majoritários (10), e limitaram-se em avaliar propriedades antimicrobianas através das concentrações inibitórias, fungicidas e bactericidas. Três foram revisões da literatura e apenas um ensaio clínico esteve presente, sendo o cegamento e a randomização ausentes. Evidenciou-se a necessidade de um melhor delineamento metodológico das pesquisas e de uma maior produção de estudos clínicos ou in vivo.

Palavras-chave: extratos vegetais, etnobotânica, fitoterapia, odontologia, plantas medicinais.

\section{INTRODUCTION}

Plants have been used worldwide with medicinal purposes. In this perspective, Brazil has a great potential for the development of herbal medicine, since it holds the largest plant diversity in the world and also encompasses a wide social diversity (Martins, 2000; Albuquerque \& Hanazaki, 2006). This might explain the resurgence of natural medicine in Brazil in recent years, with emphasis

Recebido para publicação em 26/09/2013 
on the use of medicinal plants for health recovery (Yunes et al., 2001; Borba \& Macedo, 2006). Plant extracts are not only raw materials for industrial production of pure chemical derivatives, but they are also components of herbal formulations used in the treatment of several diseases (Lima Júnior \& Souza, 2005).

The use of herbal medicines has been an alternative therapeutic option for health professionals in the search for greater pharmacological activity, higher biocompatibility, low toxicity and a more affordable treatment to the population (Castilho et al., 2007). In addition, it may be provided equivalent registered drugs with proven efficacy for the same or other clinical indications (Rates, 2001).

The increasing use of herbal medicine, commonly in a judicious and indiscriminate way, together with the scarcity of high quality studies in this field and limited access to the literature on this topic, have raised many issues about the effectiveness and safety of herbal medicines. The empirical knowledge accumulated over the years based on cultural tradition, coupled with scientific findings has shown that medicinal plants and herbal medicines bring benefits to the body, as well as can cause adverse effects and toxicity and be contraindicated (Alexander Garcia \& Simões, 2005) just as any other substance with pharmacological effects.

Although with many gaps in scientific research (Oliveira, 2010), there was an official recognition of the practicability of using herbal medicines in the Brazilian public health system (Bettega et al., 2011). In 2008, the 2.960/2008 inter-ministry ordinance implemented the National Program of Medicinal Plants and Herbal Medicines in the Unified Health System (UHS). The role of dentists in this field has gained more strength with the Resolution 082/2008 of the Federal Council of Dentistry (FCO), through which herbal medicine was recognized as an integrative and complementary practice in oral health (Assis, 2009).

Even though a number of public policies have been created with the purpose of encouraging the study and clinical use of medicinal plants, herbal medicine takes place in a modest way by dental practitioners mainly due to the lack of scientific support - specifically for plant species indicated to prevent/treat oral diseases (Oliveira, 2010). According to Bettega et al. (2011), this resistance can be attributed to the lack of standardization and knowledge about prescription as well as skepticism and conviction that manufactured drugs have more guaranteed effectiveness. Nevertheless, there has been a growing interest in this subject in recent years, demonstrated by the increased number of studies with natural products in dentistry (Castilho et al., 2007). These studies point out that medicinal plants can be used therapeutically against oral diseases (Oliveira, 2010), displaying anti-inflammatory, analgesic and anti-hemorrhagic properties for the treatment of odontalgias and other oral disorders (Lima Júnior et al., 2005).

Thus, given the need for further research addressing the use of medicinal plants for the prevention and treatment of oral diseases, this study aimed to critically analyze the scientific production on medicinal plants published in Brazilian specialized journals over the last ten years.

\section{MATERIALS AND METHODS}

\section{Search strategy}

This is a bibliographical study using the indirect documentation technique. A critical review of scientific studies about the use of herbal medicines in dentistry was carried out following the guidelines of the Brazilian Cochrane Collaboration. Searches were conducted in Brazilian specialized journals of

Table 1. Identification of inclusion and exclusion criteria defined for this study.

\begin{tabular}{cc}
\hline Inclusion criteria & Exclusion criteria \\
\hline $\begin{array}{c}\text { Period and language of publishing: Studies } \\
\text { published between January 2002 and December 2012 } \\
\text { in Portuguese and English }\end{array}$ & Studies published before 2002 \\
\hline Study design: Clinical trials and laboratory assays (in & All studies that did not match the search strategy \\
vivo, in situ and in vitro) & Studies evaluating synthetic products \\
\hline Experimental product: Naturally-occurring agents & Studies whose design did not characterize the \\
\hline Primary interest: Use against oral diseases (dental \\
caries, periodontal diseases, mycoses)
\end{tabular}


medicinal plants indexed in the database SciELO (Brazilian Journal of Pharmacognosy; Brazilian Journal of Medicinal Plants; Brazilian Journal of Pharmaceutical Sciences; Acta Botanica Brasilica). The articles discussing the use of herbal medicines to prevent and treat oral diseases, published between January 2002 and December 2012, were selected. Articles were included only from these journals in order to assess the national production through the publication of results approved by expert reviewers in the subject (Table 1).

The selection of descriptors was done based on DecS (subject descriptors in health sciences, BIREME http://www.bireme.br) in Portuguese and English. At first, it was used "herbal medicine" OR "medicinal plants" AND "Dentistry" in the SciELO database. Subsequently, more detailed searches were performed directly on each national specialized journal, using only the descriptor "dentistry," in order to avoid losses of articles that met the inclusion criteria proposed for the study. The abstracts that met the inclusion criteria regardless of the language in which they had been published were pre-selected. Then an analysis of the methodological quality of full texts was performed by four independent trained examiners.

\section{Analysis of studies}

The variables analyzed were: study nature (quantitative or qualitative), study type (descriptive or experimental), search strategy (laboratory or field research), research subjects (cell culture, animals or humans), statistical analysis, language and year of publication. Botanical data were gathered to evaluate: species and part of the plant used in the study, pharmaceutical form, phytochemical analysis and botanical identification. It was also considered the disease against which the herbal medicine was used and its therapeutic purpose. For clinical studies, it was considered: the type of subject allocation (randomization or convenience), sampling (probabilistic or non-probabilistic), masking of products, use of placebo or control, intra and interexaminer calibration, and pilot study.

In order to assess the methodological consistency of each article, an assessment of quality, internal and external validity was carried out independently for each study by four examiners. Clinical studies were classified according to Jadad's scale (Jadad 1996), which assigns a score from 0 to 5 to the studies. Jadad's scale checks the validity of the evidence on interventions and evaluates three conditions: randomization, blinding and loss of follow up. Based on these criteria, it is assigned a score to the study ranging from 0 to 5 . Articles that did not meet the inclusion criteria (addressing the use of herbal medicines in dentistry) were classified as "discarded".

\section{RESULTS}

The search strategy in the databases resulted in 155 studies (Table 2).

After abstracts were examined, 44 studies were selected to be read in full, from which 16 met the methodological criteria required (Table 3 and 4). Of these 16 articles, 10 were in vitro laboratory studies; 02 were ethnobotanical field studies based on interviews with healers or community; 03 were literature reviews; and only 01 was a phase 2 clinical trial.

\section{DISCUSSION}

The Brazilian territory is home to around $20 \%$ of global biodiversity including plants, which serve as raw material for the production of herbal and other medicines. Together with this is the vast cultural and ethnic diversity of Brazil, responsible for the knowledge on the management and use of medicinal plants passed down over generations (Brazil, 2006). Hence, national journals falling into this scope are expected to provide a large contribution with several high quality publications from different areas of health, including dentistry. Nevertheless, the results found in this study are in disagreement with this expectation.

The study types found were: in vitro (majority), ethnobotanical studies, literature review and clinical trial. Despite the remarkable importance of in vitro studies, they take place on the base of the pyramid regarding levels of scientific evidence (Cook et al., 1995). However, considering the great potential of Brazil in this area, researches with scientific evidence such as in vivo studies, clinical trials or systematic reviews were considered scarce in the field of dentistry. Although there was a large number of in vitro studies, which could lead a variety of methodological approaches, the investigations were basically limited to MIC and MBC/MFC tests of extracts against microorganisms causing dental caries (Vasconcelos et al., 2008; Silva et al., 2008; Pereira et al., 2006; Melo et al., 2006; MacedoCosta et al., 2009; Carvalho et al., 2009) and oral candidiasis (Lubian et al., 2010; Castro \& Lima, 2011; Alves et al., 2006).

Besides the antifungal and antibacterial potential of medicinal plants against dental infections, it is important to study and evaluate other properties such as anesthetic, analgesic (Soares et al., 2009), antiviral (Kazyiama et al., 2012), antimutagenic and antitumor (Maciel et al., 2002) activities. Toxicological studies addressing possible adverse effects of medicinal plants (Cowan, 1999; Veiga Júnior et al., 2005) are also scarce, given their importance for the evolution and progression of researches in this field. In this respect, it was observed that the scientific 
Table 2. Selection of studies according to the descriptors and reading of abstracts and full texts.

\begin{tabular}{|c|c|c|}
\hline \multirow{5}{*}{$\begin{array}{l}\text { Identification of potentially relevant } \\
\text { studies based on their titles and } \\
\text { abstracts }\end{array}$} & Brazilian Journal of Pharmacognosy & 107 \\
\hline & Brazilian Journal of Medicinal Plants & 13 \\
\hline & Brazilian Journal of Pharmaceutical Sciences & 33 \\
\hline & Acta Botanica Brasilica & 2 \\
\hline & Total & 155 \\
\hline \multirow{5}{*}{$\begin{array}{l}\text { Collection and reading of selected } \\
\text { studies }\end{array}$} & Brazilian Journal of Pharmacognosy & 32 \\
\hline & Brazilian Journal of Medicinal Plants & 10 \\
\hline & Brazilian Journal of Pharmaceutical Sciences & 5 \\
\hline & Acta Botanica Brasilica & 1 \\
\hline & Total & 44 \\
\hline \multirow{5}{*}{ Included studies } & Brazilian Journal of Pharmacognosy & 9 \\
\hline & Brazilian Journal of Medicinal Plants & 6 \\
\hline & Brazilian Journal of Pharmaceutical Sciences & 0 \\
\hline & Acta Botanica Brasilica & 1 \\
\hline & Total & 16 \\
\hline \multirow{5}{*}{ Excluded studies } & Brazilian Journal of Pharmacognosy & 23 \\
\hline & Brazilian Journal of Medicinal Plants & 4 \\
\hline & Brazilian Journal of Pharmaceutical Sciences & 5 \\
\hline & Acta Botanica Brasilica & 0 \\
\hline & Total & 28 \\
\hline
\end{tabular}

Table 3. Main author, language, year of publication, article title, national journal and study design of selected studies.

\begin{tabular}{|c|c|c|c|c|c|c|}
\hline Main Author & Language & $\begin{array}{c}\text { Year of } \\
\text { publication }\end{array}$ & Article title & Journal & Study Design & $\begin{array}{l}\text { JADAD } \\
\text { Scale }\end{array}$ \\
\hline ALVES, P. M. & Portuguese & 2006 & $\begin{array}{l}\text { Antifungal activity of the extract of Psidium guajava } \\
\text { Linn. ("goiabeira") upon Candida yeasts of the oral } \\
\text { cavity: an in vitro evaluation }\end{array}$ & $\begin{array}{l}\text { Brazilian Journal } \\
\text { of Pharmacognosy }\end{array}$ & $\begin{array}{c}\text { Quantitative } \\
\text { Experimental } \\
\text { in-vitro } \\
\text { Controlled }\end{array}$ & $\begin{array}{c}\text { Not } \\
\text { applicable }\end{array}$ \\
\hline MELO, A. F. M. & Portuguese & 2006 & $\begin{array}{l}\text { In vitro antimicrobial activity of an extract of Anacardium } \\
\text { occidentale L. against Streptococcus species }\end{array}$ & $\begin{array}{l}\text { Brazilian Journal } \\
\text { of Pharmacognosy }\end{array}$ & $\begin{array}{c}\text { Quantitative } \\
\text { Experimental } \\
\text { in-vitro } \\
\text { Controlled }\end{array}$ & $\begin{array}{l}\text { Not } \\
\text { applicable }\end{array}$ \\
\hline PEREIRA, J. V. & Portuguese & 2006 & $\begin{array}{l}\text { In vitro antibacterial and anti-adherent effect of the } \\
\text { extract of Punica granatum Linn. upon dental biofilm } \\
\text { microorganisms }\end{array}$ & $\begin{array}{l}\text { Brazilian Journal } \\
\text { of Pharmacognosy }\end{array}$ & $\begin{array}{c}\text { Quantitative } \\
\text { Experimental } \\
\text { in-vitro } \\
\text { Controlled }\end{array}$ & $\begin{array}{l}\text { Not } \\
\text { applicable }\end{array}$ \\
\hline OLIVEIRA, F. Q. & Portuguese & 2007 & Plants species indicated in odontology & $\begin{array}{l}\text { Brazilian Journal } \\
\text { of Pharmacognosy }\end{array}$ & $\begin{array}{l}\text { Qualitative } \\
\text { Descriptive }\end{array}$ & $\begin{array}{c}\text { Not } \\
\text { applicable }\end{array}$ \\
\hline
\end{tabular}


Table 3. Main author, language, year of publication, article title, national journal and study design of selected studies.

\begin{tabular}{|c|c|c|c|c|c|c|}
\hline \multicolumn{7}{|c|}{ continuação... } \\
\hline SILVA, M.S.A & Portuguese & 2008 & $\begin{array}{l}\text { In vitro antimicrobial activity and anti-adherence of } \\
\text { Rosmarinus officinalis Linn. against oral planktonic } \\
\text { bacteria }\end{array}$ & $\begin{array}{l}\text { Brazilian Journal } \\
\text { of Pharmacognosy }\end{array}$ & $\begin{array}{c}\text { Quantitative } \\
\text { Experimental } \\
\text { in-vitro } \\
\text { Controlled }\end{array}$ & $\begin{array}{c}\text { Not } \\
\text { applicable }\end{array}$ \\
\hline $\begin{array}{l}\text { VASCONCELOS, } \\
\text { K. R.F. }\end{array}$ & Portuguese & 2008 & $\begin{array}{l}\text { In vitro assessment of antibacterial activity of a dental } \\
\text { cement constituted of Copaifera multijuga Hayne } \\
\text { oil-resin }\end{array}$ & $\begin{array}{l}\text { Brazilian Journal } \\
\text { of Pharmacognosy }\end{array}$ & $\begin{array}{c}\text { Quantitative } \\
\text { Clinical trial } \\
\text { in-vivo } \\
\text { Controlled }\end{array}$ & $\begin{array}{l}\text { Not } \\
\text { applicable }\end{array}$ \\
\hline SANTOS, E. B. & Portuguese & 2009 & $\begin{array}{l}\text { Etnobotanical studies of medicinal plants for oral } \\
\text { conditions in the municipality of João Pessoa, Brazil }\end{array}$ & $\begin{array}{l}\text { Brazilian Journal } \\
\text { of Pharmacognosy }\end{array}$ & $\begin{array}{c}\text { Quantitative } \\
\text { Qualitative } \\
\text { Descriptive } \\
\text { Field research }\end{array}$ & $\begin{array}{l}\text { Not } \\
\text { applicable }\end{array}$ \\
\hline $\begin{array}{l}\text { M A C E D - } \\
\text { COSTA, M. R. }\end{array}$ & Portuguese & 2009 & $\begin{array}{l}\text { Effectiveness of the Myrciaria cauliflora (Mart.) O. Berg. } \\
\text { extract on oral bacteria }\end{array}$ & $\begin{array}{l}\text { Brazilian Journal } \\
\text { of Pharmacognosy }\end{array}$ & $\begin{array}{c}\text { Quantitative } \\
\text { Experimental } \\
\text { in-vitro } \\
\text { Controlled }\end{array}$ & $\begin{array}{l}\text { Not } \\
\text { applicable }\end{array}$ \\
\hline LUBIAN, C.T & Portuguese & 2010 & $\begin{array}{l}\text { Antifungal activity of the aqueous extract from Arctium } \\
\text { minus (Hill) Bernh. (Asteraceae) on oral Candida } \\
\text { species }\end{array}$ & $\begin{array}{l}\text { Brazilian Journal } \\
\text { of Medicinal Plants }\end{array}$ & $\begin{array}{c}\text { Quantitative } \\
\text { Experimental } \\
\text { in-vitro } \\
\text { Uncontrolled }\end{array}$ & $\begin{array}{l}\text { Not } \\
\text { applicable }\end{array}$ \\
\hline $\begin{array}{l}\text { OLIVEIRA, A. C. } \\
\text { M. }\end{array}$ & Portuguese & 2011 & $\begin{array}{l}\text { Use of Melaleuca alternifolia Cheel (Myrtaceae) oil } \\
\text { in dentistry: perspectives on its use as alternative } \\
\text { antimicrobial to infectious diseases of oral origin }\end{array}$ & $\begin{array}{l}\text { Brazilian Journal } \\
\text { of Medicinal Plants }\end{array}$ & $\begin{array}{l}\text { Qualitative } \\
\text { Descriptive }\end{array}$ & $\begin{array}{c}\text { Not } \\
\text { applicable }\end{array}$ \\
\hline PAIVA, L. C. A. & Portuguese & 2011 & $\begin{array}{l}\text { Clinical and laboratorial evaluation of Uncaria } \\
\text { tomentosa (Cat's Claw) gel on oral candidiasis }\end{array}$ & $\begin{array}{l}\text { Brazilian Journal } \\
\text { of Pharmacognosy }\end{array}$ & $\begin{array}{c}\text { Quantitative } \\
\text { Clinical trial } \\
\text { in-vivo } \\
\text { Controlled }\end{array}$ & 1 \\
\hline FREIRES, I.A. & English & 2012 & $\begin{array}{l}\text { Clinical applicability of natural product(s)-containing } \\
\text { mouthwashes as adjunctive treatment of biofilm- } \\
\text { induced gingivitis: a systematic review }\end{array}$ & $\begin{array}{l}\text { Brazilian Journal } \\
\text { of Medicinal Plants }\end{array}$ & $\begin{array}{c}\text { Qualitative } \\
\text { Descriptive } \\
\text { Systematic } \\
\text { review }\end{array}$ & $\begin{array}{l}\text { Not } \\
\text { applicable }\end{array}$ \\
\hline ALMEIDA, L.F.D. & English & 2012 & $\begin{array}{l}\text { Antifungal activity of essential oils against clinical } \\
\text { samples of Candida albicans isolated from HIV-positive } \\
\text { patients }\end{array}$ & $\begin{array}{l}\text { Brazilian Journal } \\
\text { of Medicinal Plants }\end{array}$ & $\begin{array}{c}\text { Quantitative } \\
\text { Experimental } \\
\text { in-vitro } \\
\text { Controlled }\end{array}$ & $\begin{array}{l}\text { Not } \\
\text { applicable }\end{array}$ \\
\hline
\end{tabular}

Rev. Bras. Pl. Med., Campinas, v.16, n.3, p.618-627, 2014. 
Table 4. Main author and year, study subjects, plant and therapeutic purpose, methods, calibration of examiners, pilot study and statistical analysis of selected studies.

\begin{tabular}{|c|c|c|c|c|c|c|c|}
\hline $\begin{array}{l}\text { Main author/ } \\
\text { Year }\end{array}$ & Study subjects & $\begin{array}{l}\text { Pharmaceutical } \\
\text { form/ part of the } \\
\text { plant }\end{array}$ & $\begin{array}{l}\text { Therapeutic } \\
\text { purpose/ } \\
\text { Pathology }\end{array}$ & Sample description & Methods & $\begin{array}{l}\text { Calibration } \\
\text { and pilot } \\
\text { study }\end{array}$ & $\begin{array}{l}\text { Statistical } \\
\text { analysis }\end{array}$ \\
\hline $\begin{array}{l}\text { ALVES, P. M. } \\
(2006)\end{array}$ & $\begin{array}{l}\text { Fungal strains: Candida } \\
\text { albicans, C. tropicalis, } \\
\text { C. stelatoidea and C. } \\
\text { krusei }\end{array}$ & $\begin{array}{l}\text { Hydroalcoholic } \\
\text { extract / Guava } \\
\text { leaves ( } P \text { sidium } \\
\text { guajava Linn). }\end{array}$ & $\begin{array}{l}\text { Treatment of } \\
\text { mycosis }\end{array}$ & $\begin{array}{l}\text { Strains of C. albicans } \\
(24), \text { C. tropicalis } \\
\text { (25), C. stelatoidea } \\
\text { (23) and C. krusei (9) }\end{array}$ & $\begin{array}{l}\text { McGinnis methods } \\
\text { (1980): Solid } \\
\text { diffusion method for } \\
\text { determination of MIC. } \\
\text { Tests were performed } \\
\text { in duplicate. }\end{array}$ & $\begin{array}{l}\text { Not } \\
\text { applicable }\end{array}$ & Descriptive \\
\hline $\begin{array}{l}\text { BORBA, A. M. } \\
(2006)\end{array}$ & Human beings & Not applicable & $\begin{array}{l}\text { Not } \\
\text { applicable }\end{array}$ & $\begin{array}{l}40 \text { people with } \\
\text { folk knowledge in } \\
\text { medicinal plants. }\end{array}$ & $\begin{array}{l}\text { Semi-structured } \\
\text { interviews and } \\
\text { observation on which } \\
\text { medicinal plant had } \\
\text { been popularly used in } \\
\text { the region. }\end{array}$ & Not stated & Descriptive \\
\hline $\begin{array}{l}\text { MELO, A. F. M. } \\
(2006)\end{array}$ & $\begin{array}{l}\text { Bacterial strains: } S \text {. } \\
\text { mutans, S. mitis and } \\
\text { S.sanguis }\end{array}$ & $\begin{array}{l}\text { Hydroalcoholic } \\
\text { extract / } \\
\text { Stem bark of } \\
\text { Anacardium } \\
\text { occidentale L }\end{array}$ & $\begin{array}{l}\text { Prevention of } \\
\text { dental caries }\end{array}$ & $\begin{array}{l}\text { Replication of three } \\
\text { standard strains: } S \text {. } \\
\text { mitis, S. mutans and } \\
\text { S. sanguis }\end{array}$ & $\begin{array}{l}\text { It was used the } \\
\text { technique of } \\
\text { wells by the agar } \\
\text { diffusion method for } \\
\text { determination of MIC. } \\
\text { Tests were performed } \\
\text { in duplicate. }\end{array}$ & $\begin{array}{l}\text { Not } \\
\text { applicable }\end{array}$ & Descriptive \\
\hline $\begin{array}{l}\text { PEREIRA, J. V. } \\
(2006)\end{array}$ & $\begin{array}{l}\text { Bacterial strains: } \\
\text { Streptococcus mitis, } \\
\text { Streptococcus mutans, } \\
\text { Streptococcus sanguis, } \\
\text { Streptococcus sobrinus, } \\
\text { and Lactobacillus casei }\end{array}$ & $\begin{array}{l}\text { Hydroalcoholic } \\
\text { extract / } \\
\text { stem bark of } \\
\text { pomegranate } \\
\text { (Punica } \\
\text { granatum Linn.) }\end{array}$ & $\begin{array}{l}\text { Prevention of } \\
\text { dental caries }\end{array}$ & $\begin{array}{l}\text { Standard strains } \\
\text { of Streptococcus } \\
\text { mitis (ATCC 9811), } \\
\text { Streptococcus mutans } \\
\text { (ATCC 25175), } \\
\text { Streptococcus } \\
\text { sanguis } \\
\text { (ATCC 10557), } \\
\text { Streptococcus } \\
\text { sobrinus (ATCC } \\
\text { 27609) Lactobacillus } \\
\text { casei (ATCC 7469) }\end{array}$ & $\begin{array}{l}\text { It was used the agar } \\
\text { diffusion method for } \\
\text { determination of MIC } \\
\text { and the technique of } \\
\text { inclined glass tubes } \\
\text { for determination } \\
\text { of the MICA } \\
\text { (Minimum Inhibition } \\
\text { Concentration of } \\
\text { Adherence) in the } \\
\text { presence of } 5 \% \\
\text { sucrose. }\end{array}$ & $\begin{array}{l}\text { Not } \\
\text { applicable }\end{array}$ & Descriptive \\
\hline $\begin{array}{l}\text { OLIVEIRA, F. Q. } \\
(2007)\end{array}$ & $\begin{array}{l}\text { Books, scientific and } \\
\text { popular articles and } \\
\text { websites }\end{array}$ & Not applicable & $\begin{array}{l}\text { Identification } \\
\text { of species } \\
\text { indicated for } \\
\text { the treatment } \\
\text { of dental } \\
\text { diseases }\end{array}$ & Not applicable & $\begin{array}{l}\text { For each species, the } \\
\text { following information } \\
\text { was gathered: scientific } \\
\text { and popular name, } \\
\text { family, pharmaceutical } \\
\text { form and therapeutic } \\
\text { purpose. }\end{array}$ & Not stated & Not stated \\
\hline $\begin{array}{l}\text { SILVA, M.S.A } \\
(2008)\end{array}$ & $\begin{array}{l}\text { Bacterial strains: } \\
\text { Streptococcus mitis, } \\
\text { Streptococcus } \\
\text { sanguinis, } \\
\text { Streptococcus mutans, } \\
\text { Streptococcus sobrinus } \\
\text { and Lactobacillus casei }\end{array}$ & $\begin{array}{l}\text { Hydroalcoholic } \\
\text { extract / leaves } \\
\text { and stems of } \\
\text { Rosmarinus } \\
\text { officinalis Linn. } \\
\text { (rosemary) }\end{array}$ & $\begin{array}{l}\text { Prevention of } \\
\text { dental caries }\end{array}$ & $\begin{array}{l}\text { Standard strains } \\
\text { of Streptococcus } \\
\text { mitis (ATCC 98811) } \\
\text { Streptococcus } \\
\text { sanguinis } \\
\text { (ATCC 10556), } \\
\text { Streptococcus mutans } \\
\text { (ATCC 25175), } \\
\text { Streptococcus } \\
\text { sobrinus (ATCC } \\
\text { 27609) and } \\
\text { Lactobacillus casei } \\
\text { (ATCC 7469) }\end{array}$ & $\begin{array}{l}\text { It was used the agar } \\
\text { diffusion method } \\
\text { for determination of } \\
\text { MIC and technique } \\
\text { of inclined tubes for } \\
\text { determination of MICA } \\
\text { in the presence of } 5 \% \\
\text { sucrose. }\end{array}$ & $\begin{array}{l}\text { Not } \\
\text { applicable }\end{array}$ & Descriptive \\
\hline $\begin{array}{l}\text { VASCONCELOS, } \\
\text { K. R. F. (2008) }\end{array}$ & $\begin{array}{l}\text { Bacterial strains: } \\
\text { Streptococcus mutans } \\
\text { and S. sanguinis. }\end{array}$ & $\begin{array}{l}\text { Oleoresin / stem } \\
\text { of Copaifera } \\
\text { multijuga Hayne } \\
\text { (copaiba) }\end{array}$ & $\begin{array}{l}\text { Treatment of } \\
\text { dental caries }\end{array}$ & $\begin{array}{l}\text { Standard strains of } \\
\text { Streptococcus mutans } \\
\text { (ATCC 25175) and } \\
\text { S. sanguinis (ATCC } \\
\text { 15300) }\end{array}$ & $\begin{array}{l}\text { Method of dilution } \\
\text { in liquid culture } \\
\text { medium by Andrews } \\
\text { (2001), modified by } \\
\text { Abinader (2005) for } \\
\text { determination of MIC. }\end{array}$ & $\begin{array}{l}\text { Not } \\
\text { applicable }\end{array}$ & Descriptive \\
\hline
\end{tabular}


Table 4. Main author and year, study subjects, plant and therapeutic purpose, methods, calibration of examiners, pilot study and statistical analysis of selected studies.

\begin{tabular}{|c|c|c|c|c|c|c|c|}
\hline $\begin{array}{l}\text { SANTOS, E. B. } \\
(2009)\end{array}$ & Human beings & Not applicable & $\begin{array}{l}\text { Prevention } \\
\text { and treatment } \\
\text { of oral } \\
\text { diseases }\end{array}$ & Not applicable & $\begin{array}{l}\text { It was carried out a } \\
\text { survey using a form. } \\
\text { The sample consisted } \\
\text { of healers ("raizeiros") } \\
(n=20) \text { and users } \\
(n=200) \text {. }\end{array}$ & Not stated & $\begin{array}{l}\text { Descriptive and } \\
\text { inferential }\end{array}$ \\
\hline $\begin{array}{l}\text { CARVALHO, C. } \\
\text { M. (2009) }\end{array}$ & $\begin{array}{l}\text { Bacterial strains: } \\
\text { Streptococcus mutans } \\
\text { Streptococcus sobrinus } \\
\text { and Streptococcus } \\
\text { sanguis }\end{array}$ & $\begin{array}{l}\text { Hydroalcoholic } \\
\text { extract / leaves } \\
\text { of jabuticaba } \\
\text { tree (Myrciaria } \\
\text { cauliflora) }\end{array}$ & $\begin{array}{l}\text { Prevention of } \\
\text { dental caries }\end{array}$ & $\begin{array}{l}\text { Standard strains of } \\
\text { Streptococcus mutans } \\
\text { (ATCC 25175), } \\
\text { Streptococcus } \\
\text { sobrinus (ATCC } \\
\text { 27609) and } \\
\text { Streptococcus } \\
\text { sanguis (ATCC } \\
\text { 10557) }\end{array}$ & $\begin{array}{l}\text { Solid medium } \\
\text { diffusion method for } \\
\text { determination of MIC. }\end{array}$ & $\begin{array}{l}\text { Not } \\
\text { applicable }\end{array}$ & Descriptive \\
\hline $\begin{array}{l}\text { MACEDO- } \\
\text { COSTA, M. R. } \\
(2009)\end{array}$ & $\begin{array}{l}\text { Bacterial strains: } \\
\text { Streptococcus } \\
\text { mitis, Streptococcus } \\
\text { mutans, Streptococcus } \\
\text { sanguinis, } \\
\text { Streptococcus oralis, } \\
\text { Streptococcus } \\
\text { salivarius, and } \\
\text { Lactobacillus casei. }\end{array}$ & $\begin{array}{l}\text { Hydroalcoholic } \\
\text { extract / leaves } \\
\text { of jabuticaba } \\
\text { tree (Myrciaria } \\
\text { cauliflora) }\end{array}$ & $\begin{array}{l}\text { Prevention of } \\
\text { dental caries }\end{array}$ & $\begin{array}{l}\text { Streptococcus } \\
\text { mitis (ATCC 903), } \\
\text { Streptococcus mutans } \\
\text { (ATCC 25175), } \\
\text { Streptococcus } \\
\text { sanguinis } \\
\text { (ATCC 15300), } \\
\text { Streptococcus oralis } \\
\text { (ATCC 10557), } \\
\text { Streptococcus } \\
\text { salivarius (ATCC } \\
\text { 7073) and } \\
\text { Lactobacillus casei } \\
\text { (ATCC 9595). }\end{array}$ & $\begin{array}{l}\text { Solid medium } \\
\text { diffusion method for } \\
\text { determination of MIC. } \\
\text { Tests were performed } \\
\text { in duplicate. }\end{array}$ & $\begin{array}{l}\text { Not } \\
\text { applicable }\end{array}$ & $\begin{array}{l}\text { Descriptive and } \\
\text { inferential }\end{array}$ \\
\hline $\begin{array}{l}\text { LUBIAN, C.T } \\
(2010)\end{array}$ & $\begin{array}{l}\text { Fungal strains: } \\
\text { Candida albicans, } \\
\text { C.tropicalis, C. glabrata, } \\
\text { C. stellatoidea, C. } \\
\text { dubliniensis and C. } \\
\text { krusei }\end{array}$ & $\begin{array}{l}\text { Aqueous extract / } \\
\text { leaves of Arctium } \\
\text { minus (Hill) } \\
\text { Bernh (burdock) }\end{array}$ & $\begin{array}{l}\text { Treatment of } \\
\text { mycosis }\end{array}$ & $\begin{array}{l}\text { Standard strains } \\
\text { (ATCC 44858) and } \\
\text { three different strains } \\
\text { of clinical isolates } \\
\text { of C. albicans, C. } \\
\text { tropicalis and C. } \\
\text { krusei }\end{array}$ & $\begin{array}{l}\text { Antifungal tests were } \\
\text { carried out using the } \\
\text { broth microdilution } \\
\text { technique. }\end{array}$ & $\begin{array}{l}\text { Not } \\
\text { applicable }\end{array}$ & Descriptive \\
\hline $\begin{array}{l}\text { CASTRO, R. D. } \\
(2011)\end{array}$ & $\begin{array}{l}\text { Fungal strains: Candida } \\
\text { albicans and } C . \\
\text { tropicalis }\end{array}$ & $\begin{array}{l}\text { Essential oil / } \\
\text { leaves of Ocotea } \\
\text { odorifera Vell. } \\
\text { (sassafras) and } \\
\text { Rosmarinus } \\
\text { officinalis L. } \\
\text { (rosemary) }\end{array}$ & $\begin{array}{l}\text { Treatment of } \\
\text { mycosis }\end{array}$ & $\begin{array}{l}\text { Strains of C. albicans } \\
\text { (ATCC- 90028), C. } \\
\text { albicans (ATCC- } \\
\text { 76615), C. albicans } \\
\text { (ATCC-76645), } \\
\text { C.albicans (ATCC- } \\
\text { 76485), C.tropicalis } \\
\text { (ATCC-13803), C. } \\
\text { albicans (LM-42V), } \\
\text { C. albicans (18F), C. } \\
\text { albicans (MD-37), C. } \\
\text { albicans (LM-968), C. } \\
\text { albicans (ICB-12), C. } \\
\text { tropicalis (LM-708), C. } \\
\text { tropicalis (LM-14), C. } \\
\text { tropicalis (LM-028), C. } \\
\text { tropicalis (LM-37), C } \\
\text { tropicalis (LM-13) and } \\
\text { C. tropicalis (LM-759). }\end{array}$ & $\begin{array}{l}\text { Microdilution } \\
\text { technique was used } \\
\text { for determination of } \\
\text { MIC according to the } \\
\text { Reference method for } \\
\text { broth dilution antifungal } \\
\text { susceptibility testing of } \\
\text { yeasts. }\end{array}$ & $\begin{array}{l}\text { Not } \\
\text { applicable }\end{array}$ & Descriptive \\
\hline $\begin{array}{l}\text { OLIVEIRA, A. C. } \\
\text { M. (2011) }\end{array}$ & $\begin{array}{l}\text { Books, scientific articles } \\
\text { and websites }\end{array}$ & Not applicable & $\begin{array}{l}\text { Prevention } \\
\text { and treatment } \\
\text { of dental } \\
\text { caries, } \\
\text { periodontal } \\
\text { disease and } \\
\text { pulp injuries. }\end{array}$ & Not applicable & $\begin{array}{l}\text { No search strategy } \\
\text { was described }\end{array}$ & Not stated & Not stated \\
\hline
\end{tabular}


Table 4. Main author and year, study subjects, plant and therapeutic purpose, methods, calibration of examiners, pilot study and statistical analysis of selected studies.

\begin{tabular}{|c|c|c|c|c|c|c|c|}
\hline $\begin{array}{l}\text { PAIVA, L. C. A. } \\
\text { (2011) }\end{array}$ & Human beings & $\begin{array}{l}\text { Phytodrug in gel } \\
\text { form contained } \\
\text { in tubes with } \\
10 \mathrm{~g} \text { of Uncaria } \\
\text { tomentosa (cat's } \\
\text { claw)* }\end{array}$ & $\begin{array}{l}\text { Treatment of } \\
\text { mycosis }\end{array}$ & $\begin{array}{l}20 \text { patients with } \\
\text { clinical and } \\
\text { laboratorial diagnosis } \\
\text { of Candida infection. }\end{array}$ & $\begin{array}{l}\text { Test group (10): gel } 3 x \\
\text { / day for } 14 \text { days. } \\
\text { Control group (10): } \\
\text { Miconazole/Daktarin } \\
\text { Gel } 3 x \text { / day for } 14 \\
\text { days. } \\
\text { Blinding was not } \\
\text { reported. }\end{array}$ & Not stated & $\begin{array}{l}\text { Descriptive and } \\
\text { inferential }\end{array}$ \\
\hline $\begin{array}{l}\text { FREIRES, I.A } \\
(2012)\end{array}$ & $\begin{array}{l}\text { Systematic review in } \\
\text { the databases Medline, } \\
\text { SciELO, LILACS and } \\
\text { Cochrane Library. }\end{array}$ & Not applicable & $\begin{array}{l}\text { Prevention } \\
\text { and treatment } \\
\text { of biofilm- } \\
\text { induced } \\
\text { gingivitis } \\
\text { using } \\
\text { mouthwashes } \\
\text { containing } \\
\text { natural } \\
\text { products. }\end{array}$ & Not applicable & $\begin{array}{l}\text { Searches were } \\
\text { carried out in the } \\
\text { databases Medline, } \\
\text { SciELO, LILACS and } \\
\text { Cochrane Library using } \\
\text { combination of the } \\
\text { key-words "gingivitis/ } \\
\text { natural products/ } \\
\text { herbal medicines/ } \\
\text { mouthwashes, in } \\
\text { English, Portuguese } \\
\text { and Spanish. } \\
\text { The methodological } \\
\text { design of studies was } \\
\text { assessed according to } \\
\text { Jadad's scale (Jadad } \\
\text { et al., 1996). }\end{array}$ & Yes & Descriptive \\
\hline $\begin{array}{l}\text { ALMEIDA, L.F.D } \\
(2012)\end{array}$ & $\begin{array}{l}\text { Fungal strains of } \\
\text { Candida albicans } \\
\text { isolated from HIV } \\
\text { patients (CA 01- CA 15) } \\
\text { and a standard strain } \\
\text { (ATCC 76845). }\end{array}$ & $\begin{array}{l}\text { Essential oils } \\
\text { / Ocimum } \\
\text { basilicum } \\
\text { L. (basil), } \\
\text { Cymbopogon } \\
\text { martinii L. } \\
\text { (palmarosa), } \\
\text { Thymus vulgaris } \\
\text { L. (thyme) and } \\
\text { Cinnamomum } \\
\text { cassia Blume } \\
\text { (Chinese } \\
\text { cinnamon) }\end{array}$ & $\begin{array}{l}\text { Treatment of } \\
\text { mycosis }\end{array}$ & $\begin{array}{l}\text { Strains of Candida } \\
\text { albicans (CA 01- CA } \\
\text { 15) isolated from } \\
\text { HIV+ patients and } \\
\text { a standard strain } \\
\text { (ATCC 76845) }\end{array}$ & $\begin{array}{l}\text { Antifungal tests were } \\
\text { carried out using the } \\
\text { broth microdilution } \\
\text { technique }\end{array}$ & $\begin{array}{l}\text { Not } \\
\text { applicable }\end{array}$ & Descriptive \\
\hline
\end{tabular}

*The plant part was not mentioned.

production on herbal medicine in dentistry needs to broaden thematic approaches and advance with regard to the level of evidence. This fact can be confirmed by the scarcity of phase 1 and 2 clinical trials, scientifically supported by previous laboratory studies.

Only one clinical trial was retrieved in the searches (Paiva et al., 2009), which was a phase 2 study investigating the effect of an herbal product on Candida-associated denture stomatitis. The authors did not report sample size calculation or any other scientific parameter to justify the number of participants recruited. Additionally, there was no description of blinding and sample randomization, but only of loss of follow-up of subjects, which led this study to reach a score 1 on Jadad's scale. This scale serves as a parameter for qualifying clinical trials, by which a study is considered biased when it does not score a value greater than 3 .

Another aspect to be considered is the fact that the selection of plants to be investigated commonly lacks satisfactory basis, as observed in the studies by Carvalho et al. (2009) and CostaMacedo et al. (2009). These authors investigated the extract from Myrciaria cauliflora (Mart.) O. Berg. (jabuticaba tree) leaves even in the absence of justifiable evidence, being only supported by the information that it contained ascorbic acid, tannins and cyaniding, and peonidin glycosides (Reynertson et al., 2006) in its composition.

Although such studies require triplicate experiments, Carvalho et al. (2009) carried out their experiment in duplicate, leaving room for the unreliability of their findings.

Ethnobotanical studies have been developed as a way to guide experts on the botanical species 
to be investigated. It allows for the identification of plants with their respective forms of use and folk medicine established over the time, according to Santos et al. (2009) and Borba \& Macedo (2006). Given the importance of this type of study, it surprises the fact that only two ethnobotanical investigations were among the national publications. Moreover, in order to contribute to further researches of this nature as well as to ensure the reliability of the data collection instrument, the authors were expected to describe in detail the form (data collection instrument) used in their study, clarifying whether it was based on some other form previously used and validated in the literature. Attaching the form used to the published article would also favor its use by other studies, whose results could be discussed and compared.

The way how researches obtained the plants to be analyzed lacks standardization and raises questions about the safety of their origin conditions. While Pereira et al. (2006), MacedoCosta et al. (2009), Carvalho et al. (2009) and Alves et al. (2006) found the plant material in street fairs, Silva et al. (2008) and Melo et al. (2006) collected their material in controlled environments, derived from plantation sites located in the university where their studies were undertaken. Lubian et al. (2010) also adopted a criterion for plant collection, as they reported it was held during the flowering period. In streets fairs or similar places, the lack of information about soil and growing environment conditions favors methodological biases. Freitas et al. (2004) pointed out that plants produce secondary metabolites as a function of an interaction with the environment, responding to chemical and biological factors. Thus, this lack of standardization can lead to biased researches as well as explain diverging results obtained in similar studies.

Some in vitro studies used percolation or leaching in continuous flow at room temperature as the extraction method (Alves et al., 2006; Pereira et al., 2006), while other authors have made use of an alcoholic or hydroalcoholic extraction solution to obtain their extracts (Silva et al., 2008; Carvalho et al., 2009; Macedo-Costa et al., 2009; Melo et al., 2009). Despite identifying the method used, its choice was not justified in the studies, which might lead to shortcomings in the methodological design. Alves et al. (2006) and Pereira et al. (2006) reported only the choice for room temperature for the leaching process because high temperatures might interfere with the ultimate stability of the substance. According to Cowan (1999), for studies to be more systematic, the methods of extraction of essential oils and also the methodological design of in vitro tests should be standardized. This would facilitate findings interpretation and comparison.
Interpretation of results and establishment of conclusions should be done avoiding inappropriate extrapolations. Oliveira et al. (2011) conducted a literature review and concluded improperly based on limited in vitro studies that TTO (tea tree oil) is an alternative agent to chlorhexidine against oral microorganisms. Indeed, conclusions and recommendations of this nature are reliable when supported by randomized controlled trials or systematic literature reviews. The latter bring together results of properly designed clinical trials, therefore assisting in clinical decisions (Cook et al., 1995).

Given the above, the present study indicates that the national production of scientific articles addressing medicinal plants or herbal medicine in dentistry is still scarce; most studies were laboratorial and investigated the antifungal and antibacterial properties of plant materials. It is important to emphasize the need for further publications in this area and also for advances in science by means of clinical trials, previously supported by in vitro findings.

The broadening of thematic approaches in relation to the national production available can bring interesting implications. Furthermore, it becomes critical to define systematic methodological standards in order to facilitate the comparison of results and avoid biases to scientific inferences.

\section{REFERENCE}

ALBUQUERQUE, U.P.; HANAZAKI, N. As pesquisas etnodirigidas na descoberta de novos fármacos de interesse médico e farmacêutico: fragilidades e pespectivas. Revista Brasileira de Farmacognosia, v.16s, p.678-89, 2006.

ALEXANDRE, R.F. et al. Fitoterapia Baseada em Evidências. Parte 1. Medicamentos Fitoterápicos Elaborados com Ginkgo, Hipérico, Kava e Valeriana. Acta farmaceutica bonaerense. v.24, n.2, p.300-09, 2005.

ALMEIDA, L.F.D. et al. Atividade antifúngica de óleos essenciais frente a amostras clínicas de Candida albicans isoladas de pacientes HIV positivos. Revista Brasileira de Plantas Medicinais. v.14, n.4, p.649-55, 2012.

ALVES, P.M. et al. Atividade antifúngica do extrato de Psidium guajava Linn. (goiabeira) sobre leveduras do gênero Candida da cavidade oral: uma avaliação in vitro. Revista Brasileira de farmacognosia. v.16, n.2 p.192-96, 2006.

ASSIS, C. Plantas medicinais na Odontologia. Revista Brasileira de Odontologia. v.66, n. 1, p.72-75, 2009.

BETTEGA, P.V.C. et al. Fitoterapia: dos canteiros ao balcão da farmácia. Archives of Oral Research. v.7, n.1, p.89-97, 2011.

BORBA, A.M.; MACEDO, M. Plantas medicinais usadas para a saúde bucal pela comunidade do bairro Santa 
Cruz, Chapada dos Guimarães, MT, Brasil. Acta Botanica Brasilic. v.20, n.4, p.771-82, 2006.

BRASIL. Ministério da Saúde. Secretaria de Ciência, Tecnologia e Insumos Estratégicos. Departamento de Assistência Farmacêutica. Política nacional de plantas medicinais e fitoterápicos. Brasília: Ministério da Saúde, 2006.

CARVALHO, C.M et al. Efeito antimicrobiano in vitro do extrato de jabuticaba [Myrciaria cauliflora (Mart.) O.Berg.] sobre Streptococcus da cavidade oral. Revista Brasileira de Plantas Medicinais. v.11, n.1, p79-83, 2009.

CASTILHO, A.R. Produtos naturais em Odontologia. Revista Saúde. v.1, n. 1p.11-19, 2007.

CASTRO, R.D. LIMA, E.O. Atividade antifúngica dos óleos essenciais de sassafrás (Ocotea odorifera Vell.) e alecrim (Rosmarinus officinalis L.) sobre o gênero Candida. Revista Brasileira de Plantas Medicinais. v.13, n.2, p.203-08, 2011.

COOK, D.J. et al. Clinical recommendations using levels of evidence for antithrombotic agents. Chest. v.108, p.227S-230S, 1995.

COWAN, M.M. Plant products as antimicrobial agents. Clinical Microbiology Reviews. v. 12, n.4 , p.564-82, 1999.

FREIRES, I.A. Clinical applicability of natural product(s)containing mouthwashes as adjunctive treatment of biofilm-induced gingivitis: a systematic review. Revista Brasileira de Plantas Medicinais. v.14,n.4, p. 700-11, 2012.

FREITAS, M.S.M. et al. Crescimento e produção de fenóis totais em carqueja [Baccharis trimera (Less.) D.C.] em resposta à inoculação com fungos micorrízicos arbusculares, na presença e na ausência de adubação mineral. Revista Brasileira de Plantas Medicinais. v.6, p.30-34, 2004.

JADAD, A.R. et al. Assessing the quality of reports of randomized clinical trials: is blinding necessary? Controlled Clinical Trials. v.17, n.1, p.1-12, 1996.

KAZIYAMA, V.M. Atividade antiviral de extratos de plantas medicinais disponíveis comercialmente frente aos herpes vírus suíno e bovino. Revista Brasileira de Plantas Medicinais.v.14, n.3, p.522-28, 2012.

LIMA JÚNIOR, J.F.; SOUZA, E.C.F. Situando a fitoterapia frente às racionalidades médicas ocidentais contemporâneas. Saúde Revista, v. 7, n.16, p.49-53, 2005.

LIMA JÚNIOR, J.F. O uso de fitoterápicos. Saúde Revista. v.7, n16, p.11-17, 2005.

LUBIAN, C.T. et al. Atividade antifúngica do extrato aquoso de Arctium minus (Hill) Bernh. (Asteraceae) sobre espécies orais de Candida. Revista Brasileira de Plantas Medicinais. v.12, n.2, p.157-62, 2010.

MACEDO-COSTA, R.M. Eficácia do extrato de Myrciaria cauliflora (Mart.) O. Berg. (jabuticabeira) sobre bactérias orais. Revista Brasileira de Farmacognosia. v.19, n.2b, p.565-71, 2009.

MACIEL, M.A.M. et al. Plantas medicinais: a necessidade de estudos multidisciplinares. Química Nova. v. 25, n.3, p.429-38, 2002.

MARTINS, E.R. et al. Plantas medicinais. Viçosa: Editora UFV, 2000.

MELO, A.F.M. et al. Atividade antimicrobiana in vitro do extrato de Anacardium occidentale L. sobre espécies de Streptococcus. Revista Brasileira de Farmacognosia. v.16, n. 2, p.202-05, 2006.

OLIVEIRA, A.C.M. et al. Emprego do óleo de Melaleuca alternifolia Cheel (Myrtaceae) na odontologia: perspectivas quanto à utilização como antimicrobiano alternativo às doenças infecciosas de origem bucal. Revista Brasileira de Plantas Medicinais. v.13, n.3, p.492-99, 2011.

OLIVEIRA, F.Q. et al. Espécies vegetais indicadas na odontologia. Revista Brasileira de Farmacognosia. v.17, n.4, p.466-76, 2007.

OLIVEIRA, M.A.C. Plantas medicinais utilizadas para problemas bucais: estudo etnobotânico em diferentes biomas da Paraíba. 2010. 111p. Trabalho de Conclusão de Curso, Universidade Federal da Paraíba, João Pessoa.

PAIVA, L.C.A. et al. Avaliação clínica e laboratorial do gel da Uncaria tomentosa (Unha de Gato) sobre candidose oral. Revista Brasileira de Farmacognosia. v.19: n.2a, p.423-28, 2009.

PEREIRA, J.V. Efeito antibacteriano e antiaderente in vitro do extrato da Punica granatum Linn. sobre microrganismos do biofilme dental. Revista Brasileira de Farmacognosia. v.16, n.1, p.88-93, 2006.

RATES, S.M.K. Promoção do uso racional de fitoterápicos: uma abordagem no ensino de Farmacognosia. Revista Brasileira de Farmacognosia. v.11, n.1, p.57-69, 2001.

REYNERTSON, K.A. Bioactive depsides and anthocyanins from Jaboticaba (Myrciaria cauliflora). Journal of Natural Products. v.69, n. , p.1228-30, 2006.

SANTOS, E.B. Estudo etnobotânico de plantas medicinais para problemas bucais no município de João Pessoa, Brasil. Revista Brasileira de Farmacognosia.v.19, n.2a, p.321-324, 2009.

SILVA, M.A.S. Atividade antimicrobiana e antiaderente in vitro do extrato de Rosmarinus offi cinalis Linn. sobre bactérias orais planctônicas. Revista Brasileira de Farmacognosia, v.18, n.2, p.236-240, 2008.

SOARES, C.C. Atividade analgésica do extrato da Pectis jangadensis (S. Moore.) Revista Brasileira de Farmacognosia, v.19, n.1, p.77-81, 2009.

VASCONCELOS, K.R.F. Avaliação in vitro da atividade antibacteriana de um cimento odontológico à base de óleo-resina de Copaifera multijuga Hayne. Revista Brasileira de Farmacognosia. v.18s, p.733-738, 2008.

VEIGA JUNIOR, V.F. et al. Plantas medicinais: cura segura? Química Nova, v.28, n.3, p.519-528, 2005.

YUNES, R.A. et al. Fármacos e fitoterápicos: a necessidade do desenvolvimento da indústria de fitoterápicos e fitofármacos no Brasil. Química Nova, v.24, n.1, p.147152, 2001. 